OPEN ACCESS

Edited by:

Veit Roessner,

University Hospital Carl Gustav

Carus, Germany

Reviewed by:

Omneya Ibrahim,

Suez Canal University, Egypt

Karen Schmeelk-Cone,

University of Rochester, United States

${ }^{*}$ Correspondence:

Deana G. Trimble

deanatrimble@gmail.com

Specialty section

This article was submitted to Child and Adolescent Psychiatry,

a section of the journal

Frontiers in Pediatrics

Received: 13 April 2021

Accepted: 23 August 2021

Published: 14 September 2021

Citation:

Trimble DG and Chandran A (2021) Associations Between Sad Feelings and Suicide Behaviors in the 2019 Youth Risk Behavior Survey: A Call for Action. Front. Pediatr. 9:694819.

doi: 10.3389/fped.2021.694819

\section{Associations Between Sad Feelings and Suicide Behaviors in the 2019 Youth Risk Behavior Survey: A Call for Action}

\author{
Deana G. Trimble ${ }^{1 *}$ and Aruna Chandran ${ }^{2}$ \\ 'Department of Health, Behavior, and Society, Johns Hopkins Bloomberg School of Public Health, Institute for Global \\ Tobacco Control, Baltimore, MD, United States, ${ }^{2}$ Department of Epidemiology, Johns Hopkins Bloomberg School of Public \\ Health, Baltimore, MD, United States
}

Purpose: Suicide is the second leading cause of death among high school aged students in the United States. Significant risk factors for suicidal behaviors among youth include diagnoses of depression or other mental illnesses. The association between self-reported sad feelings and suicidal behaviors has been understudied in the literature among United States high school students.

Methods: The 2019 National Youth Risk Behavior Survey (YRBS) school-based questionnaire, coordinated by the CDC, captured a nationally-representative sample ( $N$ $=13,677$ ) of students' responses to health-related behaviors. National sex-stratified prevalences of sad feelings and suicidal behaviors were calculated. Adjusted weighted logistic regression was used to examine the association between self-reported sad feelings and suicidal behaviors.

Results: Out of 13,677 high-school students, $35.8 \%$ of students self-reported sad feelings. Suicidal behaviors in the overall sample included $18.2 \%$ had seriously considered suicide, $15.2 \%$ made a plan on how they would attempt suicide, and $7.3 \%$ attempted suicide within the past 12 months. There was an 8-11-fold increased odds of all suicidal behaviors among those who reported sad feelings among both females and males.

Conclusions: This study reveals a remarkably high prevalence of sad feelings among US youth, and underscores a significant association between self-reported sad feelings and suicidal behaviors among this population. The YRBS survey, routinely administered across US high school students, should be better leveraged to target interventions toward these high-risk youth in order to decrease the significant burden of suicidal behaviors among adolescents.

Keywords: YRBS, youth, suicide, suicidal behaviors, mental health, sad feelings 


\section{IMPLICATIONS AND CONTRIBUTION}

This study highlights the high prevalence of sad feelings among US adolescents, and a notably strong association between sad feelings and suicidal behaviors in this population. The YRBS survey should be leveraged to target interventions toward this high-risk population in order to decrease the burden of suicide among US adolescents.

\section{BACKGROUND}

Suicide is the tenth leading cause of death in the United States, responsible for over 48,000 deaths annually $(1,2)$. Suicide mortality is particularly concerning among teenagers and young adults in the United States, with 6,211 deaths in 2018 among individuals 15-24 years of age, making it the second leading cause of death in this age group (2). The broader construct of suicidal behaviors encompasses suicide ideation (thoughts about suicide), attempted suicide (intentional self-harm with the goal of taking one's own life), and completed suicide (intentional self-harm resulting in death) (3). The 2019 Center for Disease Control's Youth Risk Behavior Survey (YRBS) showed a prevalence of suicide ideation among high school students of $18.8 \%$, and increase from $17.2 \%$ in 2017 (4). Similarly, the prevalence of attempted suicide among high school students was $8.9 \%$, an increase from $7.4 \%$ in 2017 (4). Increased prevalence of suicide ideation and attempts was noted among individuals reporting female sex, American Indian/Alaska Native, Black/African American or Mixed race, and gay/lesbian/bisexual orientation (4).

Many studies have shown mental health illnesses to be a significant risk factor for suicidal behaviors; up to $90 \%$ of adolescents who have died by suicide have a reported history of mental illness (5-8). Among adolescents, depression is the mental health diagnosis most strongly associated with suicide $(5,8)$. While the YRBS survey does not ask about diagnosis of depression, it does asks youth to report feelings of sadness or hopelessness (hereafter referred to as "sad feelings") that persist for 2 weeks. Importantly, persistent sad feelings are consistent with some of the DSM-5 diagnostic criteria for depression (9). To our knowledge, there have not been any evaluations within the YRBS of the association between self-reported sad feelings and suicidal behaviors. If self-reported sad feelings are significantly associated with suicidal behaviors, this represents a unique opportunity to identify teens that are potentially at high risk for suicidal behaviors through a survey that is routinely administered in middle and high schools across the US. Therefore, in this study we aim to quantify the association between sad feelings and suicidal behaviors using the national 2019 YRBS survey.

\section{METHODS}

\section{Data Source}

This study utilizes data from the 2019 Youth Risk Behavior Surveillance System (YRBSS) survey data, a nationallyrepresentative survey coordinated by the Centers for
Disease Control and Prevention (CDC) to monitor healthrelated behaviors of middle and high school students in the United States (10). The YRBSS has collected data since 1991 on a biennial basis and aims to track the health behaviors that contribute to the leading causes of death, disability, and social problems among United States youth (10). The data for the YRBSS is collected using anonymous self-administered questionnaires completed in school (11). Analytic weights are provided in the YRBS dataset in order to allow for inferences about the broader United States population (11).

The final sample for the 2019 data were 13,677 questionnaires collected from 136 schools across the United States (11). The response rates for the data include a $75.1 \%$ school response rate, $80.3 \%$ student response rate, and $60.3 \%$ overall response rate (11).

\section{Measures}

Sad feelings were captured using the question: "During the past 12 months, did you ever feel so sad or hopeless almost every day for 2 weeks or more in a row that you stopped doing some usual activities?" (Yes/No). Suicidal behaviors were captured across three questions: "During the past 12 months, did you ever seriously consider attempting suicide?" (considered suicide), "During the past 12 months, did you make a plan about how you would attempt suicide?" (made a suicide plan), and "During the past 12 months, how many times did you actually attempt suicide?" (attempted suicide).

Covariates included age, sex, race/ethnicity, sexual orientation, school-based bullying and cyber-bullying. Age was asked "How old are you?" (integer values from 12 or younger to 18 or older). Sex was asked "What is your sex?" (female or male). Race/ethnicity was asked as "What is your race" and "Are you Hispanic or Latino?", and recoded for responses (American Indian/Alaska Native, Asian, Black or African American, Native Hawaiian/Other Pacific Islander, White, Hispanic/Latino, Multiple-Hispanic, or Multiple-NonHispanic). Sexual orientation was captured as "Which of the following best describes you?" (Heterosexual/straight, or Gay/lesbian/bisexual/not sure). School-based bullying was captured as "During the past 12 months, have you ever been bullied on school property?" and Cyber-bullying as "During the past 12 months, have you ever been electronically bullied? (Count being bullied through texting, Instagram, Facebook, or other social media.)". Substance use behaviors were captured in ever-use questions for each of the following substances: cigarette, electronic vapor product, alcohol, marijuana, synthetic marijuana, prescription pain medicine, cocaine, inhalants, heroin, methamphetamines, ecstasy, hallucinogenic drugs, and steroids. For this analysis, reported use of any of these substances was coded as "ever use."

\section{Statistical Analysis}

Descriptive statistics showing frequencies of core sociodemographic variables for the overall population, and those reporting sad feelings, considered suicide, made a suicide plan, and attempted suicide were generated using weighted 
TABLE 1 | Descriptive statistics (weighted counts and percent) of high school students in the YRBS 2019.

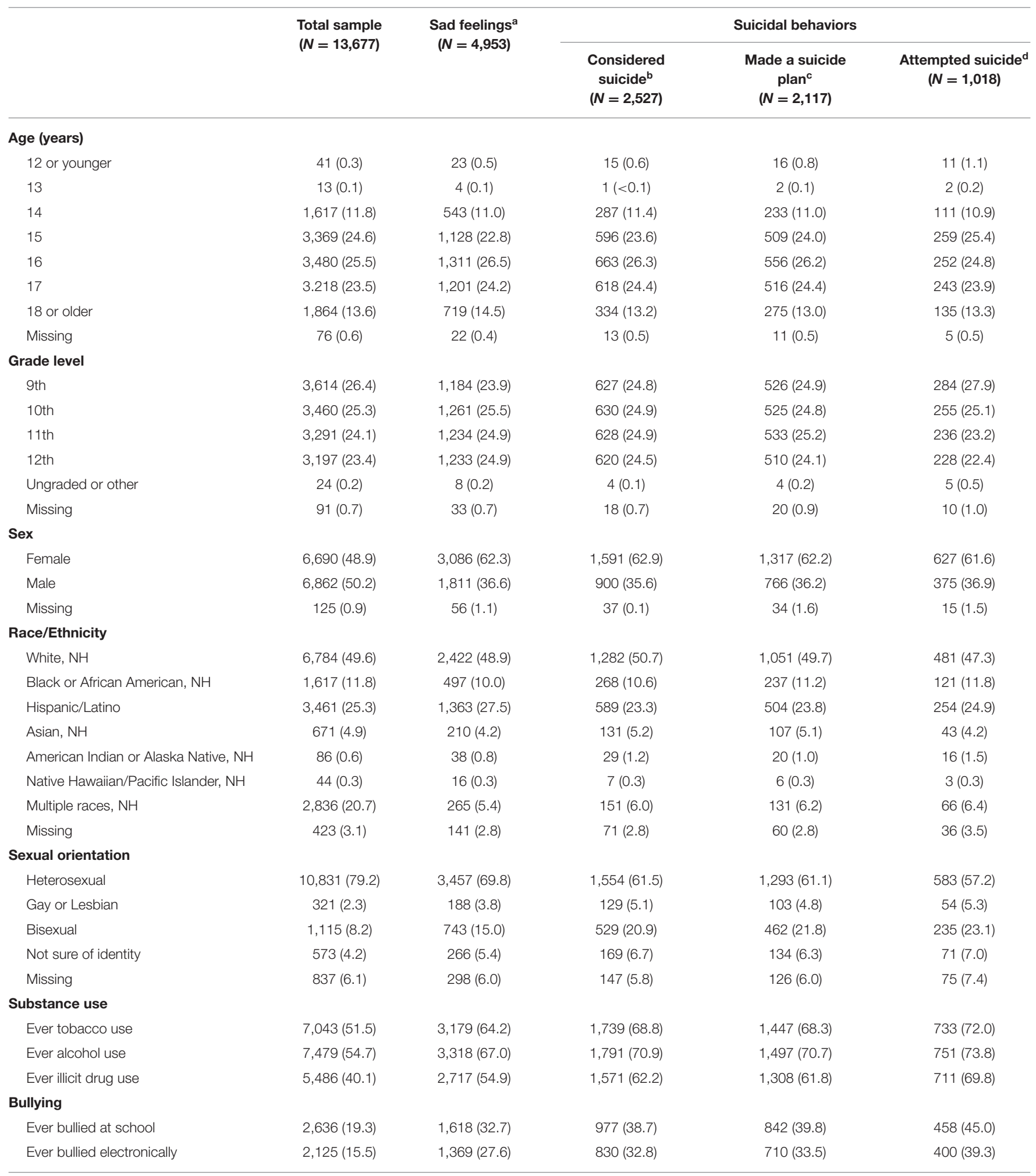

${ }^{a}$ Felt sad or hopeless for two consecutive weeks.

${ }^{b}$ Seriously consider attempting suicide during the past 12 months.

${ }^{c}$ Made a plan about how they would attempt suicide in the past 12 months.

${ }^{d}$ Actually attempted suicide in the past 12 months. 
TABLE 2 | Sex stratified prevalence (weighted counts and percent) of sad feelings, suicidal behaviors, and substance use behaviors among high school students in the YRBS 2019 ( $N=13,677)$.

\begin{tabular}{lccc}
\hline & $\begin{array}{c}\text { Females } \\
\text { number (\%) }\end{array}$ & $\begin{array}{c}\text { Males } \\
\text { number (\%) }\end{array}$ & $\begin{array}{c}\text { Pearson's } \\
\text { chi-square }\end{array}$ \\
\hline $\begin{array}{l}\text { Sad feelings } \\
\text { Suicidal behaviors }\end{array}$ & $3,086(46.1)$ & $1,811(26.4)$ & $<0.001$ \\
$\begin{array}{l}\text { Considered suicide } \\
\text { Made a suicide plan }\end{array}$ & $1,591(23.8)$ & $900(13.1)$ & $<0.001$ \\
$\begin{array}{l}\text { Attempted suicide } \\
\text { Substance use behavior }\end{array}$ & $627(9.4)$ & $766(11.2)$ & $<0.001$ \\
$\begin{array}{l}\text { Ever tobacco use } \\
\text { Ever alcohol use }\end{array}$ & $3,469(51.8)$ & $3,516(51.2)$ & $<0.001$ \\
Ever other drug use & $3,907(58.4)$ & $3,507(51.1)$ & $<.700$ \\
\hline
\end{tabular}

data. Frequencies were also stratified by sex, given the significant differences in prevalence of suicide behaviors by sex. Associations between sad feelings and each of the suicidal behaviors stratified by sex were assessed using weighted logistic regression models adjusted for the above-listed covariates. All data analysis was performed using Stata 16 (StataCorp, College Station, TX).

\section{RESULTS}

\section{Sample Demographics}

The dataset included a total unweighted sample of 13,677 high school students (Table 1). The age distribution ranged from age 12 and under to age 18 and older, with most of the students (85.4\%) falling into the $14-17$ age range. The students were similarly divided between grade levels, with $26.4 \%$ in 9 th grade, $25.3 \%$ in 10 th grade, $24.1 \%$ in 11 th grade, $23.4 \%$ in 12 th grade, and $0.2 \%$ in an ungraded or other grade. Approximately half the students were female (48.9\%) and half were male $(50.2 \%)$. The race/ethnicity distribution of students included $49.6 \%$ white (non-Hispanic), 25.3\% Hispanic/Latino, 11.8\% Black or African American (non-Hispanic), 4.9\% Asian (non-Hispanic), 0.6\% American Indian or Alaska Native (non-Hispanic), 0.3\% Native Hawaiian or other Pacific Islander (non-Hispanic), and 20.7\% multiple race (non-Hispanic) students. The majority (79.2\%) of students identified as heterosexual, and $14.7 \%$ identified as gay or lesbian, bisexual, or unsure of their sexual identity. Among all student respondents, 51.5\% reported ever tobacco use, $54.7 \%$ reported ever alcohol use, and $40.1 \%$ reported ever illicit drug use (including marijuana). Lastly, $19.3 \%$ of students reported they were bullied at school and $15.5 \%$ reported having been bullied electronically.

\section{Sex-Stratified Prevalence of Sad Feelings and Suicidal Behaviors}

Overall, $35.8 \%$ of students reported feeling sad almost every day for 2 consecutive weeks or more in a row that stopped them from doing some usual activities (Table 2). Females reported higher prevalence of sad feelings than males (46.1 vs. $26.4 \%$ ).
Nearly one-fifth of students (18.2\%) reported they seriously considered suicide in the past 12 months. Stratum-specific results showed more females considered suicide (23.8\%) than males (13.1\%). Comparing all students, 15.2 percent made a plan about how they would attempt suicide in the past 12 months. More females $(19.7 \%)$ than males $(11.2 \%)$ reported that they made a plan about how they would attempt suicide. Lastly, 7.3 percent reported they actually attempted suicide in the past 12 months. Among those who actually attempted suicide, there were more females $(9.4 \%)$ than males (5.5\%).

Of those who reported sad feelings or any of the suicidal behaviors, approximately two-thirds (63\%) were female and onethird $(36 \%)$ were male. Of those that reported substance use behavior, the prevalence was nearly 50\% female and 50\% male for tobacco use, alcohol use, and other drug use.

\section{Association Between Sad Feelings and Suicidal Behaviors}

All suicidal behaviors were significantly associated with sad feelings for both females and males (Table 3). Increases in sad feelings were associated with a nearly 10 -fold increased odds of seriously considering suicide [adjusted Odds Ratio (aOR): 9.91, 95\% Confidence Interval (CI): 7.6, 13.0] among females, and an over 11-fold increased odds among males (aOR: 11.7, 95\% CI: $8.4,16.2)$. Increases in sad/hopeless feelings increased the odds of making a suicide plan by 8.17 (95\% CI: 6.1, 11.0) among females and by 9.02 (95\% CI: 7.0, 11.7) among males. Finally, increases in sad/hopeless feelings increased the odds of suicide attempts by 9.2 (95\% CI: 5.2, 16.4) among females and 8.92 (95\% CI: $5.1,15.7$ ) among males. Of note, being bullied (both at school and electronically), substance use, and identifying as a sexual minority were also associated with a significantly increased odds of all suicide outcomes. Age group was not significant in the model, and self-identified racial category was inconsistent in its association with the outcomes.

\section{DISCUSSION}

The increasing rates of suicide among United States high school students raises major concern as it continues to remain the second leading cause of suicide in this age group $(2,12)$. The results of this study highlight the magnitude of the problem of suicide in United States as nearly one-fifth (18.2\%) of the sample reported seriously considering suicide and $7.3 \%$ reported making a suicide attempt. This result is on par with the CDC's Webbased Injury Statistics Query and Reporting System (WISQARS) results for non-fatal injuries, which estimated 5.3\% of non-fatal injuries for high school-aged youth were caused by self-harm in 2019 (13).

Understanding the potential factors that contribute to this problem is crucial to reducing the rates of these suicidal behaviors. Our study points out a $35.8 \%$ prevalence of sad feelings among high school students in the US in 2019. This is significantly higher than the $13.3 \%$ prevalence of major depressive episodes among adolescents aged 12-17 (14). Importantly, we show large (between 8 and 11 -fold increases) 
TABLE 3 | Association between feelings of sadness or hopelessness and suicidal behaviors, stratified by sex, among high school students in the YRBS $2019(\mathrm{~N}=$ 13,677).

\begin{tabular}{|c|c|c|c|c|c|c|}
\hline & \multicolumn{6}{|c|}{ Adjusted odds ratio* ( $95 \%$ Confidence interval) } \\
\hline & $\begin{array}{l}\text { Considered } \\
\text { suicide }\end{array}$ & $\begin{array}{l}\text { Made suicide } \\
\text { plan }\end{array}$ & $\begin{array}{l}\text { Attempted } \\
\text { suicide }\end{array}$ & $\begin{array}{l}\text { Considered } \\
\text { suicide }\end{array}$ & $\begin{array}{l}\text { Made suicide } \\
\text { plan }\end{array}$ & $\begin{array}{l}\text { Attempted } \\
\text { suicide }\end{array}$ \\
\hline Sad feelings & $9.91(7.6,13.0)$ & $8.17(6.1,11.0)$ & $9.20(5.2,16.4)$ & $11.7(8.4,16.2)$ & $9.02(7.0,11.7)$ & $8.92(5.1,15.7)$ \\
\hline \multicolumn{7}{|l|}{ Age (years) } \\
\hline $14-15$ & $1.29(0.3,5.6)$ & $1.04(0.2,4.1)$ & $0.09(0.0,2.5)$ & $1.94(0.4,8.7)$ & $1.11(0.2,6.3)$ & $1.67(0.3,10.9)$ \\
\hline 16 and older & $1.05(0.2,4.4)$ & $0.81(0.2,3.2)$ & $0.06(0.0,1.8)$ & $2.07(0.4,9.7)$ & $1.27(0.2,7.7)$ & $2.19(0.3,14.0)$ \\
\hline \multicolumn{7}{|l|}{ Race } \\
\hline White, $\mathrm{NH}$ & Ref & Ref & Ref & Ref & Ref & Ref \\
\hline Black, $\mathrm{NH}$ & $1.23(0.9,1.6)$ & $1.49(1.2,1.9)$ & $2.36(1.6,3.6)$ & $0.82(0.6,1.2)$ & $0.96(0.5,1.7)$ & $1.53(0.9,2.4)$ \\
\hline Hispanic/Latino & $0.87(0.7,1.1)$ & $1.03(0.8,1.3)$ & $1.35(0.9,2.1)$ & $0.71(0.5,0.9)$ & $0.69(0.5,0.9)$ & $0.79(0.5,1.2)$ \\
\hline Heterosexual & Ref & Ref & Ref & Ref & Ref & Ref \\
\hline Gay/Lesbian/Bisexual/Unsure & $2.77(2.3,3.4)$ & $2.72(2.2,3.3)$ & $2.07(1.6,2.7)$ & $2.80(2.0,4.0)$ & $2.22(1.6,3.0)$ & $3.14(2.2,4.5)$ \\
\hline Substance use & $2.34(1.7,3.2)$ & $2.08(1.6,2.7)$ & $2.44(1.7,3.6)$ & $1.89(1.4,2.5)$ & $1.70(1.3,2.2)$ & $1.65(1.2,2.3)$ \\
\hline \multicolumn{7}{|l|}{ Bullying } \\
\hline Ever bullied at school & $1.65(1.3,2.1)$ & $1.55(1.2,2.0)$ & $1.74(1.4,2.2)$ & $1.82(1.2,2.7)$ & $1.77(1.3,2.5)$ & $1.76(1.1,2.9)$ \\
\hline Ever bullied electronically & $1.33(1.1,1.6)$ & $1.45(1.1,1.9)$ & $1.56(1.2,2.1)$ & $1.45(1.0,2.0)$ & $1.49(1.0,2.1)$ & $1.86(1.2,2.9)$ \\
\hline
\end{tabular}

${ }^{*}$ Adjusted for age, sexual orientation, race/ethnicity, substance use, bullying at school and electronic bullying.

Bold denotes statistically significant associations $(p<0.05)$.

statistically significant positive associations between self-reported sad feelings and suicidal behaviors among both female and male high school students in the United States. These findings emphasize previous research in demonstrating the relationship between diagnosed mental illness and suicidal behaviors (57 ), and provide unique insight on the impact of self-reported feelings of sadness or hopelessness. These unique findings highlight the need for action as the magnitude of the association between sad feelings and suicidal behaviors is high, regardless of formal mental health diagnoses. Since students readily indicated their feelings in this survey, there is a clear need for help in this demographic.

Previous research examining sex differences in suicidal behavior in this population has shown that females are more likely to engage in suicidal behaviors, but males have higher suicide mortality $(15,16)$. Our study underscores this in that more females reported suicide ideation, suicide planning and attempted suicide. Notably, we show that the relationship between sad feelings and suicide ideation, planning and attempted suicide was actually higher for males. This association is important given what is known about sex differences in suicide behaviors; our study suggests that associations between sad feelings and suicide outcomes are similar among male and female youth. This suggests that interventions to promote discussions about sad feelings should be prioritized among both males and females.
This study presents several limitations. Firstly, the data used in this study from the 2019 YRBS was cross-sectional; therefore, we cannot draw conclusions about the causal link between sad feelings and suicidal behaviors. Second, this survey relies on self-report measures from adolescents, which could result in under- or over-reporting bias especially for those questions pertaining to sad feelings and suicidal behaviors. Finally, the YRBS questionnaire does not include questions for two important potential confounders. The questionnaire used has no measure of socioeconomic status, which has been associated with suicidal behaviors among youth (17). Additionally, the questionnaire only asks for the students' sex, and does not ask about gender identity, which has also been associated with suicidal behaviors among youth (18).

Our study strengthens existing research connecting sad or hopeless feelings to suicidal behaviors. With such a high prevalence of sad feelings among US high school students and a remarkably high magnitude of the relationship between sad feelings and suicidal behaviors, these study findings underscore the need for action in this demographic. The YRBS is performed nationally every 2 years, providing every local jurisdiction with school-level data on feelings of sadness and suicidal behaviors. These data can then inform targeted interventions for students experiencing these feelings which could ultimately decrease the number of suicidal behaviors in this population. We call on all 
local jurisdictions to leverage this routinely collected data to better address poor mental health among US teens.

\section{DATA AVAILABILITY STATEMENT}

Publicly available datasets were analyzed in this study. This data can be found here: https://www.cdc.gov/healthyyouth/data/yrbs/ data.htm; https://www.cdc.gov/healthyyouth/data/yrbs/files/ 2019/XXH2019_YRBS_Data.dat.

\section{REFERENCES}

1. Heron M. Deaths: Leading Causes for 2017. National Vital Statistics Reports vol 68 no 6. Hyattsville, MD: National Center for Health Statistics (2019).

2. National Institute of Mental Health. Mental Health Information, Statistics, Suicide. Available online at: https://www.nimh.nih.gov/health/statistics/ suicide.shtml (accessed October 1, 2020).

3. Crosby AE, Ortega L, Melanson C. Self-directed Violence Surveillance: Uniform Definitions and Recommended Data Elements, Version 1.0. Atlanta, GA: Centers for Disease Control and Prevention, National Center for Injury Prevention and Control (2011).

4. Centers for Disease Control and Prevention (CDC). 1991-2019 High School Youth Risk Behavior Survey Data. Available online at: http://yrbs-explorer. services.cdc.gov/ (accessed October 5, 2020).

5. Fleischmann A, Bertolote JM, Belfer M, Beautrais A. Completed suicide and psychiatric diagnoses in young people: a critical examination of the evidence. Am J Orthopsychiatry. (2005) 75:676-83. doi: 10.1037/0002-9432.75.4.676

6. Bachmann S. Epidemiology of suicide and the psychiatric perspective. Int $J$ Environ Res Public Health. (2018) 15:1425. doi: 10.3390/ijerph15071425

7. Waldrop AE, Hanson RF, Resnick HS, Kilpatrick DG, Naugle AE, Saunders BE. Risk factors for suicidal behavior among a national sample of adolescents: implications for prevention. J Traum Stress. (2007) 20:86979. doi: 10.1002/jts.20291

8. Shaffer D, Gould MS, Fisher P, Trautman P, Moreau D, Kleinman M, et al. Psychiatric diagnosis in child and adolescent suicide. Arch Gen Psychiatry. (1996) 53:339-48. doi: 10.1001/archpsyc.1996.01830040075012

9. Tolentino JC, Schmidt SL. DSM-5 criteria and depression severity: implications for clinical practice. Front Psychiatry. (2018) 9:450. doi: 10.3389/fpsyt.2018.00450

10. Centers for Disease Control and Prevention. Youth Risk Behavior Surveillance System (YRBSS) Overview. Available online at: cdc.gov/healthyyouth/data/yrbs/overview.htm (accessed October 5, 2020).

11. Centers for Disease Control and Prevention. methodology of the youth risk behavior surveillance system--2019. MMWR. (2013) 69:1-27.

12. Hedegaard H, Curtin SC, Warner M. Increase in Suicide Mortality in the United States, 1999-2018. NCHS Data Brief, no 362. Hyattsville, MD: National Center for Health Statistics (2020).

13. Centers for Disease Control and Prevention. WISQARS Data Visualization Tool, Non-fatal Injuries, 2019, Ages 15-19. Available online at: https://wisqars-viz.cdc.gov:8006/non-fatal/explore/selected-years?nf=eyJpbn RlbnRzIjpbIjAiXSwibWVjaHMiOlsiMzAwMCJdLCJ0cmFmZmljIjpbIjAi

\section{AUTHOR CONTRIBUTIONS}

DT: conceptualization, data curation, formal analysis, investigation, methodology, project administration, writing the original draft, and reviewing and editing. AC: data curation, formal analysis, methodology, project administration, supervision, and reviewing and editing. All authors contributed to the article and approved the submitted version.

XSwiZGlzcCI6WyIxIiwiMiIsIjQiLCI1Il0sInNleCI6WyIxIiwiMiIsIjMiXSwi YWdIR3JvdXBzTWluIjpbIjE1LTE5Il0sImFnZUdyb3Vwc01heCI6WyIxNS 0xOSJdLCJjdXN0b21BZ2VzTWluIjpbIjAiXSwiY3VzdG9tQWdlc01heCI6 WyIxOTkiXSwiZnJvbVllYXIiOlsiMjAxOSJdLCJ0b1llYXIiOlsiMjAxOSJd LCJhZ2VidXR0biI6IjVZciIsImdyb3VwYnkxIjoiQUdFR1AifQ\%3D\%3D (accessed February 12, 2021).

14. National Institute of Mental Health. Mental Health Information, Statistics, Major Depression. Available online at: https://www.nimh.nih.gov/health/ statistics/major-depression.shtml (accessed February 4, 2021).

15. Cha CB, Franz PJ, Guzmán ME, Glenn CR, Kleiman EM, Nock MK. Annual research review: suicide among youth - epidemiology, (potential) etiology, and treatment. J Child Psychol Psychiatry. (2018) 59:46082. doi: $10.1111 /$ jcpp. 12831

16. Cash SJ, Bridge JA. Epidemiology of youth suicide and suicidal behavior. Curr Opin Pediatr. (2009) 21:613-9. doi: 10.1097/MOP.0b013e32833063e1

17. Lewis SA, Johnson J, Cohen P, Garcia M, Velez CN. Attempted suicide in youth: its relationship to school achievement, educational goals, and socioeconomic status. J Abnorm Child Psychol. (1988) 16:45971. doi: 10.1007/BF00914175

18. Kuper LE, Adams N, Mustanski BS. Exploring cross-sectional predictors of suicide ideation, attempt, and risk in a large online sample of transgender and gender nonconforming youth and young adults. LGBT Health. (2018) 5:391-400. doi: 10.1089/lgbt.2017.0259

Conflict of Interest: The authors declare that the research was conducted in the absence of any commercial or financial relationships that could be construed as a potential conflict of interest.

Publisher's Note: All claims expressed in this article are solely those of the authors and do not necessarily represent those of their affiliated organizations, or those of the publisher, the editors and the reviewers. Any product that may be evaluated in this article, or claim that may be made by its manufacturer, is not guaranteed or endorsed by the publisher.

Copyright $\odot 2021$ Trimble and Chandran. This is an open-access article distributed under the terms of the Creative Commons Attribution License (CC BY). The use, distribution or reproduction in other forums is permitted, provided the original author(s) and the copyright owner(s) are credited and that the original publication in this journal is cited, in accordance with accepted academic practice. No use, distribution or reproduction is permitted which does not comply with these terms. 